
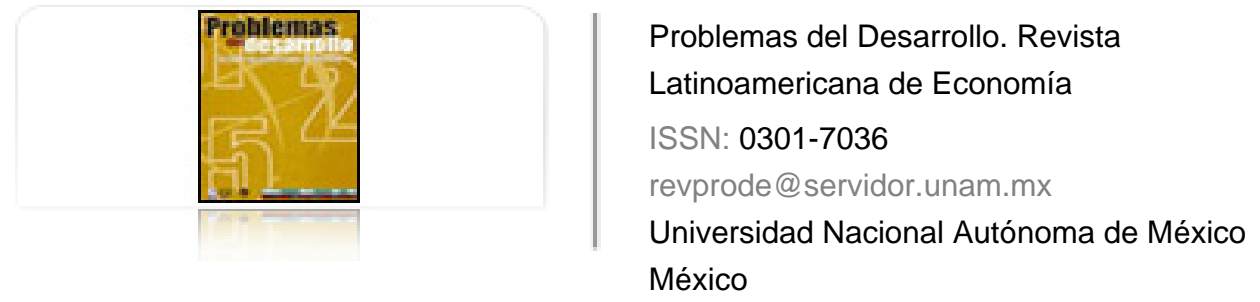

Soto Esquivel, Roberto; Correa Vázquez, Eugenia

Modelos de crisis y el uso de los instrumentos financieros derivados Problemas del Desarrollo. Revista Latinoamericana de Economía, vol. 39, núm. 155, octubre-

diciembre, 2008, pp. 11-27

Universidad Nacional Autónoma de México

Distrito Federal, México

Disponible en: http://www.redalyc.org/articulo.oa?id=11820123002

Cómo citar el artículo

- Número completo

- Más información del artículo

Página de la revista en redalyc.org

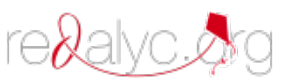

Sistema de Información Científica

Red de Revistas Científicas de América Latina, el Caribe, España y Portugal Proyecto académico sin fines de lucro, desarrollado bajo la iniciativa de acceso abierto 


\title{
MODELOS DE CRISIS Y EL USO DE LOS INSTRUMENTOS FINANCIEROS DERIVADOS
}

\author{
Roberto Soto Esquivel* \\ Eugenia Correa Vázquez**
}

Fecha de recepción: 11 de abril de 2008. Fecha de aceptación: 20 de agosto de 2008.

\section{Resumen}

Con el rompimiento de Bretton Woods, los mercados financieros se han vuelto más inestables y volátiles, lo que ha provocado escenarios de crisis más recurrentes, cada vez más profundas. Los teóricos ortodoxos han buscado predecir este tipo de crisis por medio de los llamados modelos de primera, segunda y tercera generación, pero están carentes del planteamiento, del análisis y de la interpretación de los instrumentos financieros derivados, los cuales pueden modificar el comportamiento de las variables fundamentales e interferir en el desempeño de la autoridad monetaria y financiera así como en el cumplimiento de sus objetivos.

Palabras clave: modelos, crisis financieras, innovación financiera, desregulación y mercados de derivados.

\section{Summary}

With the ending of Bretton Woods, the financial markets became more unstable and volatile, and this led to more recurrent crisis scenarios that have become increasingly profound. Orthodox theorists have sought to predict this type of crisis by means of the so-called first, second and third generation models, but they lack the formulation of analysis and interpretation of financial derivatives, which can modify the behavior of the fundamental variables and interfere with the performance of the monetary and financial authorities, as well as the fulfillment of their objectives.

Key words: models, financial crises, financial innovation, deregulation and derivatives markets.

* Profesor de asignatura B definitivo de la Facultad de Economía de la Universidad Nacional Autónoma de México. Correo electrónico: robertos@ servidor.unam.mx.

** Profesora titular C de la Facultad de Economía de la Universidad Nacional Autónoma de México. Correo electrónico: correa@ servidor.unam.mx,

Agradecemos las sugerencias y comentarios de los dictaminadores. La responsabilidad como siempre es sólo de los autores. 


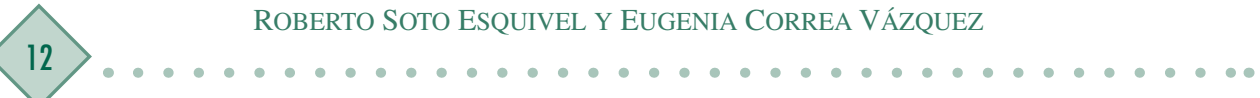

\section{Résumé}

Avec la rupture des accords de Bretton woods, les marchés financier sont devenus plus instables et volatiles, ce qui a provoqué des scénarios de crise plus récurrentes et chaque fois plus profondes. Les théoriciens orthodoxes ont cherché à prévoir ce type de crises au moyen des modèles dits de première, seconde et troisième génération, mais il manque à ceux-ci la prise en compte, l'analyse et l'interprétation des instruments financiers dérivés, lesquels peuvent modifier le comportement des variables fondamentales et interférer dans l'action de l'autorité monétaire et financière ou encore l'empêcher d'atteindre ses objectifs.

Mots clés: modèles, crises financières, innovation financière, dérégulation et marchés de dérivés.

\section{Resumo}

Com o rompimento de Bretton Woods, os mercados financeiros viraram mais instáveis e voláteis, o que provocou cenários de crises mais recorrentes, cada vez mais profundas. Os teóricos ortodoxos tentaram predizer este tipo de crise a través dos chamados modelos de primeira, segunda e terceira geração, mas estes estão carentes do planteamento, da análise e interpretação dos instrumentos financeiros derivados, os quais podem modificar o comportamento das variáveis fundamentais e interferir no desempenho da autoridade monetária e financeira, assim como o cumprimento dos seus objetivos.

Palavras chave: modelos, crises financeiras, inovação financeira, desregulação $e$ mercados de derivados. 


\section{Introducción}

$\mathcal{L}$ partir del proceso de desregulación e innovación financiera y con el desarrollo

4 de una nueva ingeniería financiera, los mercados se han vuelto más inestables y con periodos de elevada volatilidad. La creciente liquidez en estos mercados desregulados alivia el estrés y las crisis financieras, pero a su vez provoca nuevos escenarios de crisis en regiones y países así como quiebras en conglomerados financieros y no financieros. Estos procesos se han presentado con mayor frecuencia e intensidad en los últimos años y sus efectos son cada vez más profundos, en particular cuando las pérdidas se socializan. Las teorías más ortodoxas han buscado predecir los escenarios de estrés y crisis para tratar de disminuir las consecuencias de una crisis, para ello han desarrollado una serie de modelos que buscan anticiparse a los escenarios adversos. Modelos con este propósito solamente pueden alcanzar su meta en tanto sean capaces de incorporar las variables explicativas de manera certera. Esta aproximación al estudio y predicción de las crisis financieras requiere del análisis del comportamiento de variables observables con restricciones intrínsecas al propio modelo, lo que significa que tendrán variables endógenas, es decir, variables cuyo comportamiento se explica dentro de las especificaciones del modelo. Mientras que, por otro lado, se presentan variables exógenas que son explicadas fuera del modelo y permiten en conjunto encontrar una solución óptima. En general, estos modelos carecen en su planteamiento para el análisis e interpretación de los instrumentos financieros derivados, los cuales están modificando de forma muy importante el comportamiento de las variables fundamentales e interfieren en el desempeño de los instrumentos al alcance de la autoridad financiera y en el cumplimiento de sus objetivos. Es importante analizar los efectos que pueden tener las innovaciones financieras y en general la ingeniería financiera en la estabilidad y en la inestabilidad en la materia, pues se soslaya en los modelos que buscan explicar las crisis en este análisis.

Sin embargo, estos instrumentos han sido parte del agravamiento de diversas crisis como fueron la mexicana (1994-1995), la asiática (1997), la quiebra de empresas como Enron, World Com (2001/2002) y recientemente la crisis hipotecaria y crediticia en Estados Unidos (2007-2008).

Se debe insistir que este trabajo no busca desarrollar un nuevo modelo de crisis que considere este tipo de innovaciones, sino más bien destacar, al ser el objetivo central del mismo, los efectos que tiene el mercado de derivados en el comportamiento de las variables monetarias y financieras como son el tipo de cambio y la tasa de interés y que pueden ser un factor muy importante de una crisis financiera. Se pone énfasis en que los modelos actuales de crisis financieras no retoman en su análisis

Vol. 39, núm. 155, octubre-diciembre / 2008

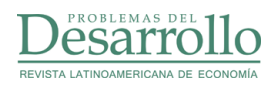


este tipo de variables, lo que los limita mucho en su trabajo de predicción de crisis, dada las condiciones que hoy imperan en los mercados de derivados.

Para ello el trabajo se divide en tres secciones, la primera retoma el análisis de los modelos de crisis e inestabilidad financiera; la segunda destaca la magnitud de los instrumentos derivados en un contexto de desregulación financiera, al considerar el caso del mercado estadounidense, y en la última parte se analiza cómo los instrumentos financieros derivados pueden influir en la determinación de variables fundamentales como son el tipo de cambio y la tasa de interés, provocando inestabilidad y crisis financiera. Sobresale que los modelos de crisis omiten por completo la existencia de los instrumentos financieros derivados en su análisis de predicción de crisis.

\section{Modelos de crisis e inestabilidad financiera: de primera,} segunda y tercera generación, así como el de Calvo-Reinhart

\section{a) Modelos de primera generación}

Los modelos de primera generación analizan los efectos en los componentes de la balanza de pagos de las economías como resultado de una incompatibilidad entre la política cambiaria de un gobierno y su política fiscal y monetaria (Agenor et al., 1992:357). Los modelos iniciales de primera generación parten de la idea de que el tipo de cambio de una economía se hace insostenible debido a una expansión del crédito interno, lo que da lugar en un inicio a una reducción de sus reservas internacionales y luego a un ataque especulativo contra la moneda doméstica, lo que obliga al banco central a abandonar la defensa del tipo de cambio para dejar que la moneda flote libremente.

La crisis de balanza de pagos se asocia con grandes movimientos en la cuenta corriente, al producirse en el periodo anterior grandes déficit en ésta por una incompatibilidad entre las políticas monetaria, fiscal y cambiaria.

La idea principal de estos modelos es que la política de tipo de cambio fijo o semifijo es insostenible si las políticas fiscal y monetaria no se sujetan a los objetivos en materia cambiaria; para ello la política monetaria debe ser restrictiva.

Estos modelos afirman que los aumentos del déficit público y oferta monetaria deben ser menores que las variaciones del tipo de cambio (en caso de ser un tipo de cambio fijo es cero, y si es un sistema crawling peg ${ }^{1}$ sería constante y positivo).

1 Régimen de tipo de cambio deslizante.

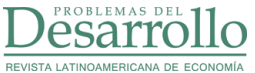


Los modelos de primera generación concluyen que la política cambiaria se vuelve insostenible debido a la fuerte expansión del crédito interno ocasionado por la monetización del déficit fiscal; ello provoca una disminución en el nivel de reservas internacionales del banco central. Antes de que éstas lleguen a cero ocurre un ataque especulativo, por lo que el banco central abandona su régimen de cambio fijo para pasar a uno flotante.

Se sostiene que se puede predecir el momento del ataque especulativo, el cual ocurre cuando el tipo de cambio esperado de flotación o tipo de cambio sombra (TCS) (the shadow floating rate) es igual al tipo de cambio fijo (TCF).

Existen diversas vertientes que buscan predecir una crisis, entre las que se destacan:

\section{Modelo de Krugman (1979)}

Krugman afirmaba que una crisis de balanza de pagos se presenta cuando existe una pérdida rápida en las reservas del banco central, lo que provoca un ataque especulativo contra su moneda, por lo que la autoridad monetaria no puede mantener el tipo de cambio (consistente en mantener un ancla con respecto a una o más monedas extranjeras y que suele ser la de su principal socio comercial o con respecto a una canasta de monedas) y se ve obligada a devaluar. El organismo bancario adopta esta medida para tratar de eliminar el riesgo en el tipo de cambio implícito en las transacciones financieras y comerciales que se realizan con el exterior.

\section{Modelo de Robert Flood y Peter Garber (1984)}

Los autores desarrollan dos modelos para estudiar el momento en el que se produce la caída de un régimen de tipo de cambio fijo. Desarrollan un modelo lineal en el que introducen una variable estocástica y después lo amplían con el concepto de esterilización de la pérdida de reservas.

En el primer modelo se parte de la hipótesis de previsión perfecta y permite identificar el momento en que se produce el decaimiento del régimen, a partir de los movimientos observados en las reservas del banco central.

El segundo modelo es uno estocástico que permite conocer la probabilidad de que se produzca un debilitamiento del régimen, así como determinar la devaluación que se producirá del tipo de cambio.

Algunas variantes a este tipo de modelos son presentadas por Connolly y Taylor (1984), quienes analizan un régimen crawling peg, en sustitución del tipo de cambio 
fijo; en tanto que Edwards (1989) considera los patrones de apreciación de la moneda y el deterioro de la cuenta corriente que suelen presentarse antes de un proceso devaluatorio.

\section{b) Modelos de segunda generación}

Los modelos de segunda generación consideran que en toda economía existe una interrelación entre el comportamiento del sector privado y las decisiones que toma el sector público o gobierno.

Por lo que una crisis financiera puede ocurrir cuando los agentes privados pronostican una devaluación de la moneda; ello puede ocurrir cuando el tipo de interés comienza a elevarse. Aunque esta situación motiva un alza en los rendimientos en los activos denominados en moneda nacional frente a la extranjera, el sostenimiento de elevadas tasas puede llevar al banco central a devaluar, presionado por la elevación del costo del servicio de la deuda. Al contrario, si los agentes privados no tienen expectativas de que se vaya a modificar la política de cambio de la moneda nacional, el tipo de interés se mantiene bajo y la devaluación es menos probable. Los modelos de segunda generación analizan variables concretas que obligan al abandono de ese cambio, identificando un proceso de retroalimentación que puede incluir un sinnúmero de factores.

En esta línea se encuadran las propuestas de Obstfeld (1994 y 1996), quien desarrolla modelos de crisis de balanza de pagos en los que incluye nuevas variables como son las tasas de interés y el nivel de crecimiento de los salarios e introduce la idea de que son las expectativas de devaluación del mercado las que inician el proceso que concluye con la devaluación de la moneda.

Modelos de contagio

Los últimos estudios se han centrado en los efectos de contagio como la "chispa" de las crisis financieras que están teniendo lugar en los últimos tiempos. El primer desarrollo que trata esta cuestión es el presentado por Gerlach y Smets (1995). En su modelo consideran dos países (A y B) con relaciones comerciales importantes, donde una depreciación de la moneda en el país A contagia al país B mediante diferentes canales: (1) incremento de la competitividad de las exportaciones de mercancías del país A, lo que produce un déficit comercial en el país B y una gradual disminución de las reservas internacionales de su banco central; (2) una reducción en el precio de las importaciones del país B conduce a una disminución en la inflación y en la demanda de dinero nacio- 
nal por parte de los residentes, lo que genera un incentivo para cambiar moneda nacional por moneda extranjera, lo que reduce las reservas del banco central.

\section{c) Modelos de tercera generación}

Con la crisis asiática de 1997-1998, el análisis ortodoxo puso en evidencia que los modelos de primera y segunda generación no daban una explicación acertada de lo sucedido en la región. Los países involucrados antes de la crisis presentaban un monto alto de reservas internacionales y un importante superávit fiscal, y aunque se consideraba que tenían focos negativos en el sistema financiero, éstos eran "conocidos y normales".

Dada la complejidad de esta crisis, se realizaron nuevos estudios de modelos teóricos que intentaran explicar estos fenómenos económicos, por lo que surgieron los modelos de tercera generación, con tres aproximaciones teóricas:

1. El primer enfoque fue desarrollado por Aghion (2001) y Krugman (1979), quienes se centraron en los problemas del balance de las empresas y en la determinación de la capacidad de inversión derivada de estos factores. Ellos sostenían que la crisis era resultado de un shock ampliado, a lo que Bernanke y Gertler (1989) y Bernanke et al. (1999) denominaron mecanismo acelerador financiero. Una depreciación real puede contraer la producción con efectos colaterales en los agentes, con lo que persiste y se amplia el shock.

2. El segundo enfoque fue desarrollado por Corsetti et al. (1998) y Mckinnon (1998); en sus estudios se destaca el crecimiento excesivo del crédito debido al riesgo moral motivado por las garantías implícitas de la autoridad. Es decir, hay un incremento de la inversión, crédito externo y el déficit en cuenta corriente; a medida que los acreedores extranjeros quieran prestar a los agentes domésticos, si se considera que existe un prestamista de última instancia en caso de contingencia. Esta situación conduce al financiamiento de proyectos poco rentables y a una escasez de capital refinanciada por el préstamo extranjero.

El déficit público no necesita ser demasiado elevado antes de la crisis, aunque la negativa de los prestamistas internacionales a refinanciar las pérdidas acumuladas obliga al gobierno a actuar y a garantizar el cumplimiento de las obligaciones de la deuda externa. Para satisfacerlo, el gobierno debe tomar medidas como reformas estructurales, en especial una política fiscal que garantice recursos suficientes para las contingencias. Así, el aumento de las expectativas de un financiamiento inflacionario provoca la caída de la moneda.

Vol. 39, núm. 155, octubre-diciembre / 2008

DeSaArrollo 
3. Los modelos que resultan de la combinación de pánicos bancarios y aspectos self-fulfilling (autorrealizables) fueron desarrollados por Chang y Velasco (1999 y 2000). En ellos se considera que una falta de liquidez en el sistema bancario es una condición necesaria y suficiente para la existencia de una crisis.

Se parte del análisis de Diamond y Dybvig, (1983) que consideran relevante el papel del banco cuando existen problemas de liquidez de los depositantes.

\section{d) Modelo Calvo-Reinhart}

A partir del reconocimiento explícito o implícito de la existencia de relaciones monetarias internacionales jerarquizadas y del desarrollo de economías con un doble patrón monetario, la explicación de las crisis financieras ha sido explorada desde diferentes perspectivas teóricas. En especial a partir de los últimos años, cuando en los diversos escenarios de crisis el tipo de cambio de las monedas locales frente a la moneda hegemónica aparece como un precio sobre el que es posible estabilizar por medio de la política económica.

Incluso se discute ampliamente en la literatura más actual no sólo las modalidades de política cambiaria que más se ajusta al modelo de economía abierta, sino también aquéllas de sustitución monetaria, políticas de dolarización y abandono del patrón monetario local.

En la aproximación teórica de Guillermo Calvo la dolarización ofrecería a las economías de mercado emergente una opción — viable y más compatible con las leyes del mercado - frente a los controles de capital (Calvo y Reinhart, 1999:13).

Estos autores discuten la conveniencia de la dolarización plena al señalar que las economías emergentes suelen comenzar con una dolarización parcial (Calvo 1999:3), la cual eleva el costo de la inestabilidad del tipo de cambio y provoca la intervención del banco central en el mercado de divisas para contrarrestar las fluctuaciones de tipo de cambio nominal. Sin embargo, si se adopta la dolarización total, ésta podría no eliminar los problemas del sector bancario pero sí los aliviaría al disminuir los creados por los desequilibrios entre las monedas y los vencimientos, al reducir los ataques especulativos contra la moneda (Calvo y Reinhart, 1999:15).

Las críticas a la dolarización, señalan estos autores, se originan en que podría existir una pérdida del derecho de señoriaje y carecer de un prestamista de última instancia (Calvo, 1999:7), pero afirman que al adoptarse este tipo de política, la pérdida del señoriaje puede compensarse con la estabilidad cambiaria alcanzada, mientras que una gran parte de las reservas internacionales podría utilizarse para prestar servicios de prestamista de última instancia (Calvo y Reinhart, 1999:15).

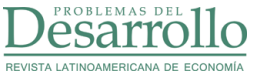


Calvo y Reinhart añaden que en un contexto de tipo de cambio fijo pero ajustable, cuando ocurre algún choque externo, se presenta un escenario de crisis pues las economías emergentes pierden acceso a los mercados internacionales de capital, se originan problemas con el cumplimiento de los pagos de la deuda externa, se incrementan las tasas de interés y disminuyen los flujos de capital, lo que puede conducir, y de hecho así ha sido, a la insolvencia o la disminución de la productividad del capital, lo que distorsiona los precios relativos y provoca quiebras masivas.

En su opinión, el manejo de la crisis se vuelve complejo puesto que la recesión económica subsiguiente a la crisis no puede ser aliviada con políticas monetarias fiscales expansionistas que disminuyan los efectos del descenso de la inversión pero que aumentan el nivel de riesgo y la inestabilidad financiera. Además se considera poco viable recurrir a políticas de controles de los movimientos internacionales de capital pues las economías emergentes sustentan sus políticas en la liberalización financiera.

Para Calvo y Reinhart (1999) se provoca una disminución en el producto interno y se incrementan los problemas en el sistema financiero y bancario cuando se presenta una interrupción repentina de los flujos de capital.

Por tanto, dicho descenso en los flujos tienen consecuencias ampliamente contraccionistas, y las recesiones así generadas son prolongadas y de lenta recuperación. Consideran también que existen algunas evidencias empíricas que revelan que es importante analizar la naturaleza —entendida ésta como su composición y volumen - que deben tener los flujos de capital, puesto que poseen diferentes determinaciones acerca de su permanencia en los mercados emergentes. De manera que estos flujos en general responden a la orientación de la política interna en los países receptores.

Por otra parte, se postula que el régimen cambiario de libre flotación si bien no evita la salida o entrada masiva de capitales, hace posible que otras políticas contribuyan a su estabilidad. Estos autores señalan las siguientes críticas a los regímenes de control de cambios y de intervención esterilizada:

a) Controles de capital. Éstos alteran la composición de los flujos de capital, reducen la proporción de flujos a corto plazo y de cartera e incrementan la inversión extranjera directa.

b) Intervención esterilizada. Esta política eleva el volumen de flujos de capital en razón de los montos a corto plazo. Esta medida podría constituir un argumento en contra de un tipo de cambio fijo pero ajustable (Calvo y Reinhart, 1999:14).

Vol. 39, núm. 155, octubre-diciembre / 2008

$\underline{\text { DeSRarrollo }}$ 
Además se sostiene que aquellas políticas de tipo de cambio influenciadas por la tasa de interés originan graves problemas de deuda externa en el corto plazo, por lo que se recomienda no utilizar la intervención esterilizada en los periodos donde haya entrada de capitales, pues provoca deuda de corto plazo en los agentes privados.

Sin embargo, los autores reconocen que bajo la libre flotación la intervención sucia o la fijación del tipo de cambio, cuando la deuda de corto plazo es mayor que las reservas internacionales, es muy probable que se produzca una crisis de balanza de pagos.

En el caso de la dolarización parcial de los pasivos, la situación puede ser más grave con un tipo de cambio fijo en la medida en que la libre flotación permite a los inversionistas nacionales evitar los préstamos en divisas, pues el riesgo monetario es mayor que con un tipo de cambio fijo (Calvo y Reinhart, 1999:15).

Así, en todo caso los autores se pronuncian en favor de un régimen cambiario de flotación, pues consideran que es menos perjudicial que el régimen de tipo de cambio fijo, aunque puede causar dificultades financieras en el mediano plazo. Sin embargo, el temor de las autoridades e inversionistas a la libre flotación y la falta de disciplina inducen a las autoridades a adoptar otras medidas como un doble tipo de cambio y controles sobre la movilidad de capital (Calvo y Reinhart, 1999:15).

En este sentido, los autores mencionan que es importante destacar lo que la teoría tradicional dice sobre la elección de un régimen de tipo de cambio, la cual debe considerar las perturbaciones: si éstas son reales en su mayor parte, el tipo de cambio tiene que flotar, de lo contrario debe fijarse (Calvo y Reinhart, 1999:15).

Estas ideas de Calvo y Reinhart fueron por muchos años en América Latina las que dominaron los criterios y políticas con que se enfrentaban las crisis financieras.

\section{Instrumentos derivados y desregulación financiera}

La importancia de los productos derivados es fundamental, es decir, hablamos de un mercado global [tanto organizado - futuros y opciones- como OTC (over the counter, fuera de bolsa) — swaps, forwards y derivados de crédito-] que a valor nocional ${ }^{2}$ representó en 1989 unos 3.8 billones de dólares y para 2007 alcanzó los casi 680 billones de dólares, lo que significa más de 10 veces el producto mundial (véase gráfica 1).

2 Valor establecido en el contrato que no necesariamente es negociado, pero sí marca un referente en el volumen de recursos que se manejan en este mercado y que es capaz de distorsionar los precios de los activos financieros y no financieros. 
Gráfica 1

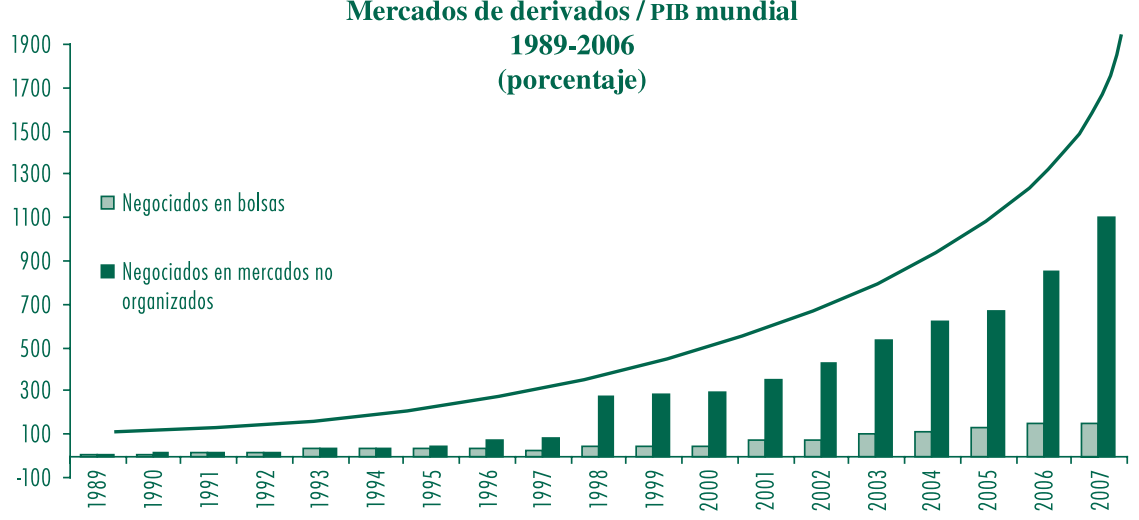

Fuente: Bank for International Settlements. International Banking and Financial Market Developments, varios años

Estos mercados se han desarrollado de forma importante a partir de la década de los ochenta del siglo pasado, aunque existen antecedentes del manejo de derivados desde la historia antigua. Sin embargo, la utilización moderna de los instrumentos derivados creció y penetró profundamente los mercados financieros desde mediados de los ochenta y de manera generalizada en los años noventa del siglo pasado. El mercado estadounidense y los mayores conglomerados financieros - con su matriz en ese país tales como Banker Trust, Chase Manhattan y otros- estuvieron entre los pioneros en la utilización contemporánea de estos instrumentos (Partnoy, 2003).

Más de 10 grandes conglomerados financieros (bancos comerciales y de inversión) incursionaron de manera creciente en la innovación financiera en la búsqueda de ampliar sus negocios, sin afectar directamente los requerimientos de capital, en un mercado caracterizado por un lento y más concentrado crecimiento de la demanda de financiamiento para la inversión y con sucesivas crisis de sobreendeudamiento que limitan la renovación y expansión del crédito.

En el cuadro 1 podemos observar que los cinco conglomerados más grandes de Estados Unidos concentran más de $96 \%$ del negocio con derivados y casi el mismo porcentaje en derivados de crédito, eso quiere decir que el grado de concentración y su exposición al riesgo es para considerarse y tomar las medidas pertinentes.

En realidad, los instrumentos derivados inicialmente conocidos se hicieron cada vez más variados y ahora son utilizados en operaciones más complejas y opacas, además de beneficiarse de las innovaciones tecnológicas en el procesamiento de la información y las comunicaciones. Ha sido en el curso de los últimos meses, a partir de la 
Cuadro 1

Conglomerados financieros

Estados Unidos

Billones de dólares

2008*

\begin{tabular}{lccc}
\hline & & \multicolumn{2}{c}{ Derivados totales' } \\
\cline { 3 - 4 } & Activos totales & Totales & De crédito \\
\hline JP Morgan Chase & 1.62 & 89.80 & 8.20 \\
CitiGroup & 2.19 & 41.30 & 3.90 \\
Bank of America & 1.70 & 38.90 & 3.08 \\
Wachiova Bank & 0.81 & 4.80 & 0.47 \\
HSBC North American Holdings & 0.49 & 4.20 & 1.30 \\
& & & \\
25 conglomerados & 11.44 & 185.90 & 17.08 \\
\hline
\end{tabular}

Fuente: Comproller of the Currency Administrator of National Banks, 2008

${ }^{1}$ El total corresponde a valor nocional

* La información corresponde al primer trimestre de 2008.

crisis hipotecaria estadounidense de 2007, que estos instrumentos están mostrando la fragilidad detrás de su amplia difusión. Es muy importante destacar la magnitud de estas transacciones debido a que superan con mucho al valor que poseen en activos totales, por lo que la burbuja especulativa es de dimensiones inimaginables.

El mercado y los instrumentos derivados han sido estudiados desde la perspectiva de la administración e ingeniería financieras y existe una muy amplia literatura. También han sido analizados desde la perspectiva de las necesidades del registro contable, de su valuación e incluso desde del derecho económico y mercantil.

Para ellos el estudio de los derivados puede tener algunos objetivos como: a) llevar a cabo operaciones de cobertura de riesgo provocados por variaciones en un activo financiero o no financiero denominado subyacente, b) determinar precios de forma eficiente, llegando a un equilibrio de acuerdo con las fuerzas de la oferta y la demanda, y c) diseminar precios por medio de las bolsas de futuros y opciones; debido a su grado de apalancamiento implícito es fácil adquirirlos.

Sin embargo, desde la visión de la ciencia económica, es decir, desde la perspectiva de la macroeconomía y en especial desde la macroeconomía poskeynesiana todavía son un objeto de estudio en exploración.

Estos instrumentos no han sido tomados en cuenta en los análisis de los modelos de crisis financieras, aunque pueden provocar efectos macroeconómicos y manipular las variables fundamentales como son el tipo de cambio, la tasa de interés (es decir, 
los efectos en la formación de precios en el mercado financiero) y el volumen de la liquidez en diferentes segmentos de los mercados financieros.

El crecimiento exponencial en el mercado organizado y no organizado de derivados puede tener consecuencias sobre el nivel de apalancamiento de los intermediarios financieros y los costos de este fondeo, lo que repercute sobre el nivel de precios fundamentales como el tipo de interés y la tasa de cambio y en ese sentido sobre la estabilidad del mercado.

En otras palabras, los productos derivados propician y alimentan la formación de burbujas especulativas, debido a que pueden modificar los precios relativos de los activos financieros y no financieros, con lo que se pueden generar olas de inflación financiera, inestabilidad y crisis. Dichas burbujas han sido un factor esencial en la concentración del excedente a lo largo del mundo económico crecientemente global.

Debemos considerar que de las crisis presentadas a lo largo de la historia, cada una de ellas con su peculiaridad, algunas son de balanza de pagos, otras bancarias y unas más financieras. Pero a partir del proceso de desregulación y liberalización financiera el uso de derivados toma mayor impulso. Es decir, cuando las variables como tipo de cambio y tasa de interés comienzan a fluctuar se incrementa en mayor medida la especulación con estas variables, y el vehículo de esta especulación son los instrumentos financieros derivados. Existe una relación entre desregulación, especulación de derivados y crisis, las cuales se retroalimentan, por lo que generan cada vez una burbuja financiera de mayor fuerza y de efectos impredecibles.

\section{Modelos de crisis financiera con presencia de innovación financiera. El caso de productos de derivados}

Una vez analizados de forma breve cada uno de los modelos de crisis financiera se retomarán algunos puntos donde se vuelven pocos funcionales bajo la presencia de productos derivados, los cuales modifican el comportamiento de las variables monetarias y financieras que son utilizadas en los modelos.

Por ejemplo, en los modelos de primera generación se estudian los efectos que se tienen en la balanza de pagos por la incompatibilidad de la política cambiaria con respecto a las políticas fiscal y monetaria.

Es decir, una política de tipo de cambio fijo o semifijo no se puede sostener si las políticas monetaria y fiscal no consideran los objetivos de la política cambiaria, lo que provocaría una crisis de balanza de pagos.

El mercado de derivados puede generar un ataque especulativo contra la moneda doméstica que tenga como consecuencia una devaluación. Si algún inversionista

Vol. 39, núm. 155, octubre-diciembre / 2008

Dessarroullo 
institucional realiza operaciones con opciones sobre el tipo de cambio que alteren su movimiento ello provocaría pérdidas importantes en el nivel de reservas internacionales, con lo que se abandonaría el tipo de cambio fijo y se aceleraría el riesgo de una crisis. Tal como lo destaca Kregel (1998), cuando analiza el papel de los instrumentos derivados que causaron inestabilidad en los mercados, provocando volatilidad en los flujos de capital, así como pérdidas generadas por la transferencia del riesgo, lo que motivó en gran medida la crisis asiática.

En cuanto a los modelos de segunda generación que están basados en las expectativas que los agentes tienen sobre el tipo de cambio y la tasa de interés, es evidente que aquellos que participan en el mercado de derivados pueden tener expectativas diferentes a aquellos que no intervienen. Los conglomerados financieros y no financieros son las principales contrapartes en el mercado de derivados, éstos pueden modificar determinados precios de los activos subyacentes, lo que provoca variaciones importantes en otros precios del mercado financieros como son básicamente la tasa de interés y el tipo de cambio.

Es más, al considerar el volumen de las operaciones y el monto que se maneja, con pequeñas variaciones se pueden obtener grandes ganancias y así crearse un mercado especulativo más importante en detrimento del sector real de la economía, contradiciendo el conjunto de la política económica de un gobierno (ya sea monetaria, cambiaría o fiscal).

Los modelos de tercera generación constan de tres enfoques, el primero basado en el balance de las empresas, el cual recibe el impacto de las depreciaciones de la moneda, lo que afecta su capacidad de pago. Esta condición de default puede provocar un efecto adverso sobre otras variables como el ingreso y la inversión, lo que afecta al resto de la economía. Ello se complica aún más si consideramos al mercado de derivados, pues se puede desencadenar una quiebra en empresas que aparentemente mostraban cierta "salud financiera". El segundo enfoque es muy interesante porque destaca que una crisis puede originarse por el excesivo crédito, tanto interno como externo. Las empresas que utilizan derivados pueden mejorar sus estados financieros, lo que les permite obtener calificaciones crediticias altas, por lo que el acceso al crédito es rápido. Pero si las condiciones del mercado cambian la situación se revierte y se provoca una quiebra significativa que puede o no alcanzar a precipitar cambios en los precios de los activos financieros y una crisis sistémica.

El tercer enfoque está relacionado con la falta de liquidez del sistema bancario. Aquí podemos resaltar que a pesar de que por medio de los derivados de crédito se puede trasladar el riesgo de una institución a otra, sea financiera o no financiera, el 
riesgo de un credit-crunch bajo condiciones adversas puede ser magnificado mediante el mercado de derivados, pues los derivados distribuyen el riesgo pero no lo desaparecen.

\section{Conclusión}

Los modelos de crisis financiera no prevén que los instrumentos derivados pueden acelerar la volatilidad tanto en el tipo de cambio como en las tasas de interés (que son los principales subyacentes que se negocian tanto en el mercado doméstico como en el ámbito internacional). Con ello repercuten tanto en la balanza de pagos como en el balance de las empresas y el nivel de liquidez del sistema bancario. Esto provoca modificaciones sustanciales en las políticas monetaria, cambiaria y fiscal de la autoridad e inhabilitan los instrumentos al alcance de los bancos centrales en condiciones de una crisis sistémica.

La razón de ser de los derivados financieros es la incertidumbre, sin ella no tiene sentido su utilización. En un principio los grandes bancos comerciales utilizaban dichos instrumentos para cubrirse de las volatilidades que existían en los subyacentes (commodities, tipos de cambio y tasas de interés), comenzado a operarse en los mercados organizados, donde existían ciertas regulaciones de la autoridad financiera. Pero conforme más avanza la innovación, los intermediarios financieros se dieron cuenta de que también podían utilizarlos para incrementar ganancias, disminuir u ocultar pérdidas, evadir y eludir regulaciones y diseminar los riesgos de sus actividades financieras. Así comienzan a tomar auge las operaciones fuera de balance, crece el mercado OTC y su contabilidad se hace crecientemente compleja tanto por su valuación como por su lugar en los balances, por lo que las regulaciones de estas actividades son prácticamente nulas. En realidad, se plantea que funciona como un mercado autorregulado, donde los participantes fijan sus propias reglas avaladas en cierta forma por las autoridades.

Ello permite que se comience a modificar la administración de los balances de las instituciones financieras y no financieras, que se adaptan a las nuevas formas de operar existentes en el mercado.

Los participantes observaron que dado el volumen a valor nocional que manejan, pueden influir en el comportamiento del precio de los subyacentes, con lo que:

a) obtienen ventajas al cerrar los contratos al influir en el precio final del subyacente,

b) los bancos centrales pierden control sobre la determinación de las variables monetarias y financieras y por tanto se dificulta el manejo de la política monetaria, y

Vol. 39, núm. 155, octubre-diciembre / 2008

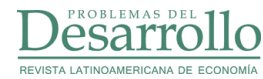




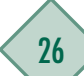

ROBERTO SOTO ESQUIVEL Y EUGENIA CORREA VÁZQUEZ

c) aparecen sucesivos escenarios de crisis, con una concentración aún mayor de los beneficios y liquidación de otros intermediarios

Por ello, es necesario preguntarse ¿es posible prever algún escenario de crisis donde estén relacionados los productos derivados? Para empezar ningún modelo de crisis financiera prevé un escenario con presencia de derivados, y al tener un manejo tan opaco se dificulta su contabilidad. Los megaconglomerados financieros y no financieros utilizan la contabilidad creativa y el valor hipotético a futuro, crean empresas para fines específicos, manejan instrumentos que ni los propios directivos de éstas conocen debido a su grado de complejidad, por tanto es muy difícil imaginar un escenario donde se considere evitar una crisis bajo presencia de derivados.

Entonces, ¿es posible regularlos? Si consideramos el escenario de incertidumbre e inestabilidad del sistema económico, los derivados se alimentan en su presencia, por lo tanto mientras se mantenga este ambiente difícilmente se podrán regular. Sin embargo, es necesaria una verdadera transparencia de estos instrumentos entre los intermediarios y todos los participantes, sobre todo la transparencia de su contabilidad, porque la gran mayoría opera en el mercado OTC. La autoridad financiera en el ámbito internacional podría replantear su papel en estos momentos, porque si se logra generar un ambiente de certidumbre y estabilidad económica, el uso de derivados perderá fuerza. Pero mientras ello ocurre es necesario estabilizar las principales fuentes de la fragilidad financiera estadounidense, transparentar las pérdidas y contener la propagación al resto de los mercados, así como intentar frenar las más agudas consecuencias de este tsunami financiero. 


\section{Bibliografía}

Agenor, Pierre-Richard et al., "Speculative Attacks and Models of Balance of Payments Crises", en IMF Staff Papers, núm 39, Washington DC, 1992, pp. 357-394.

Aghion, P. et al., "Currency Crises and Monetary Policy in an Economy with Credit Constraints" en European Economic Review, Amsterdam, vol. 45, núm. 7, 2001.

Bernanke, B. y Gertler, M., "Agency costs, net worth, and economic fluctuations", en American Economic Review, núm. 79 , Pittsburgh, 1989, pp. 14-31.

et al., "The financial acelerator in a quantitative Business Cycle Framework", en J. Taylor y M. Woodford, Handbook of macroeconomics, California, North-Holland, 1999.

Calvo, Guillermo, On dollarization, 1999, disponible en http://www.bsos.umd.edu.

y Carmen Reinhart, "Capital flow reversals, the exchange rate debate, and dollarization" en Finance and Development, Washington, Banco Mundial, septiembre de 1999.

Chang, R. y Velasco, A., "Balance Sheets and Exchange Rate Policy" en mimeo, Department of Economics, Nueva York, Nueva York University, 2000.

, "Liquidity crises in emerging markets: theory and policy", en NBER working paper, núm. 7272, Cambridge, 1999.

Connolly, Michael B.; y Taylor, Dean, "The Exact Timing of the Collapse of an Exchange Rate Regime and its Impact on the Relative Price of Traded Goods", en Journal of Money, Credit and Banking, Ohio, núm. 16, 1984, pp. 194-207.

Corsetti, G., Pesenti, P. y Roubini, N., "What caused the Asian currency and financial crisis?" en NBER working paper, núm. 6843, Cambridge, 1998.
Diamond, D. y Dybvig, P., "Bank Runs, Deposit Insurance, and Liquidity", en Journal of Political Economy, núm. 91, Chicago, 1983, pp. 401-419.

Edwards, Sebastian, Real Exchange Rates, Devaluation and Adjustment: Exchange rate Policy in Developing Countries, Cambridge, MIT Press, 1989.

Flood, Robert, y Garber, Peter, "Collapsing Exchange Rate Regimes: Some Linear Examples", en Journal of International Economics, núm. 17, Madison, Wisconsin, 1984, pp. 1-13.

Gerlach, Stefan and Frank Smets, "Contagious Speculative Attacks", en European Journal of Political Economy, núm. 11, Chicago, 1995, 45-63.

Kregel, Jan, "Derivatives and global capital flows: Applications to Asia", en Working Paper, núm. 286, EU, The Jerome Levy Economics Institute, agosto de 1998.

Krugman, Paul, "A Model of Balance of Payments Crises", en Journal of Money, Credit and Banking, núm. 11, Ohio, 1979, pp. 311-325.

Mckinnon, R., "The Overborrowing Syndrome: Are East Asian Countries Different?", en Managing Exchange Rates and Capital Flows: Lessons from the Pacific Basin, Reuven Glick, ed., Nueva York, Cambridge University Press, 1998.

Obstfeld, Maurice, "The Logic of Currency Crises", en NBER working paper, núm. 4640, Cambridge, 1994.

"Models of Currency Crises with Self-fulfilling Features" en European Economic Review, núm. 40, Amsterdam, 1996, pp. 1037-1047.

Partnoy, Frank, Codicia contagiosa, Buenos Aires, El Ateneo, 2003, 510 pp. 